\title{
EDITORIAL
}

\section{Cobertura universal de salud}

\section{Universal health coverage}

\author{
Olier-Castillo Doris, ${ }^{1}$
}

Hace pocos días, el 12 de diciembre se celebró por primera vez el Día Mundial de Cobertura de Salud, que contó con el apoyo de la Organización Panamericana de la Salud/Organización Mundial de la Salud (OPS/OMS) y muchas organizaciones de más de un centenar de países. Dicha campaña, tiene el propósito de fomentar la conciencia sobre la importancia del acceso en todos los países a la atención de salud como derecho humano básico fundamental.

"La cobertura universal de salud es un concepto y un movimiento poderoso que surgió no solo en América Latina y el Caribe, sino también a nivel mundial", dijo la Directora de la OPS/OMS, Carissa F. Etienne. "Es un proceso, en vez de una meta finita, pero es esencial para promover la salud como derecho humano y como componente crucial del desarrollo" (1).

Es una paradoja que haya que "inventar" esta campaña para uno de los derechos fundamentales de todos los seres humanos. Esto quiere decir, como todos sabemos, que muchos gobiernos tienen grandes falencias para poder garantizar este derecho a sus ciudadanos. Ya la OMS había publicado en su página web que "En muchos países, millones de personas pasan apuros porque no tienen acceso a la asistencia sanitaria que necesitan o porque el tener que costear los servicios de salud los empuja a la pobreza" (2).

Lo anterior es congruente con la OPS cuando afirma que "actualmente, en América Latina y el Caribe el $30 \%$ de la población no tiene acceso a la atención de salud por motivos económicos y el $21 \%$ no busca atención debido a barreras geográficas" (3).

Para buscar una posible solución a ello, en la 53ํㅜ reunión del Consejo Directivo de la OPS en la que participan los ministros de salud de las Américas, y Colombia estuvo representada por la Directora de Epidemiología y Demografía, Martha Lucía Ospina Martínez (4), se debatieron unos desafíos relacionados con la cobertura universal y se estableció una estrategia y plan de acción regional que según la directora de la OPS "refleja la solidaridad panamericana, ya que hemos llegado a un acuerdo basado en valores profundamente compartidos y lecciones históricamente compartidas", consideró y aclaró que alcanzar el acceso y la cobertura universal de salud es un

\footnotetext{
${ }^{1}$ Bacterióloga, Magister en Ciencias Básicas Biomédicas. Editora revista Ciencia y Salud Virtual. Facultad de Ciencias de la Salud, Corporación Universitaria Rafael Núñez, Cartagena - Colombia.
} 
"proceso gradual, y cada país seguirá su propio camino" (3).

Esperemos que este nuevo plan de acción regional que se estableció hace apenas dos meses, sea implementado a cabalidad en nuestro país, y que en un futuro nada lejano los colombianos no nos quejemos del servicio de salud, no oigamos hablar más del paseo de la muerte, sino que todos podamos festejar el Día Mundial de Cobertura de Salud, porque tenemos acceso a los servicios de salud cuando los necesitamos, lo que necesitamos y sin el temor de acudir porque no contamos con el dinero suficiente para acceder al servicio.

\section{REFERENCIAS}

1. Organización Panamericana de la Salud. El primer Día de la Cobertura Universal de Salud urge a la "salud para todos, en todas partes" Disponible en:

http://www.paho.org/hq/index.php?option=com content\&view=article\&id=10278:firsteveruniversal-health-coverage-day-urges-health-for-all-everywhere\&ltemid=1926\&lang=es

2. Organización Mundial de la Salud. Diez datos acerca de la cobertura sanitaria universal. Disponible en: http://www.who.int/features/factfiles/universal health coverage/es/

3. Organización Panamericana de la Salud. Con un histórico acuerdo sobre acceso y cobertura universal de salud, concluye el $5^{\circ}$ - Consejo Directivo de la OPS. Disponible en: http://www.paho.org/hq/index.php?option=com content\&view=article\&id=10066\&ltemid=2\&l ang=es

4. República de Colombia, Ministerio de Salud. Nueve puntos se debatirán en Consejo Directivo de la OPS. Disponible en: http://www.minsalud.gov.co/Paginas/Nueve-puntossedebatir\%C3\%A1n-en-Consejo-Directivo-de-la-OPS.aspx. 